\title{
Empirical analysis of the maximum asymptotic bias of location estimators for fuzzy number-valued data
}

\author{
Beatriz Sinova ${ }^{\mathrm{a}, *}$, Stefan Van Aelst ${ }^{\mathrm{b}}$ \\ ${ }^{a}$ Department of Statistics and O.R. and D.M., University of Oviedo, 33007 Oviedo, Spain \\ ${ }^{b}$ Department of Mathematics, KU Leuven, 3001 Leuven, Belgium
}

\begin{abstract}
Several robust measures to summarize the central tendency of a random fuzzy number have been recently proposed and compared empirically to analyze differences in finite sample behaviour. Although there is no uniformly most appropriate location estimator as in the classical settings, it seems that the empirical results are more influenced by the distributions considered for the generation of the sample data than by other aspects such as the sample size. The aim of this work is to go more deeply into the empirical comparison by investigating the maximum asymptotic bias of the location estimators, which is considered to be the most accurate measure of robustness for point estimators.
\end{abstract}

Keywords:

fuzzy number, location measure, maximum asymptotic bias, simulation 2010 MSC: 62G35, 62-07

\section{Introduction}

Fuzzy numbers suitably capture the imprecision that underlies a wide variety of real-life experiments in a mathematical way. They are especially useful when the interest is focused on data associated with human valuations, such as ratings, judgments, opinions or perceptions. De la Rosa de Sáa et al. [1] explain that the use of fuzzy numbers in these situations provides natural and very rich data descriptions, in contrast to the use of a precise scale,

\footnotetext{
* Corresponding author

Email address: sinovabeatriz@uniovi.es (Beatriz Sinova)
} 
due to the essentially imprecise nature of this kind of data. Adaptations of several statistical techniques such as regression analysis, clustering, principal components, etc., to the fuzzy-valued setting have been proposed in the literature. Nevertheless, they are mostly based on the use of the Aumann-type mean [2], which generalizes the concept of mean of a real-valued random variable. Unfortunately, the Aumann-type mean inherits the lack of robustness of the real-valued mean. Hence, outliers, errors and small data changes have a large impact on its computation. Therefore, statistical conclusions may be invalidated.

In order to guarantee that the conclusions of a study remain valid even if there are some 'contaminated observations' among the collected data, robust location measures for fuzzy number-valued data sets have been proposed (see e.g. [3, 4, 5, 6, 7, 8]). Fuzzy trimmed means, Huber and Hampel fuzzy M-estimates, the 1-norm median and the wabl/ldev/rdev median have been empirically compared by means of their bias, variance and mean squared error in [9]. As in the classical settings, it has been shown that there is no uniformly optimal location estimator. The obtained results have shown that the distributions chosen for the non-contaminated and contaminated data seem to have a larger impact on the estimates than size of the generated samples. In general, fuzzy M-estimators of location behave well for small contamination levels whereas the fuzzy trimmed means behave well when the proportion of contamination is increased, although this distinction is not as evident, for instance, when the generated fuzzy numbers use a referential interval (which means that the support of all the fuzzy numbers is contained in such an interval) and an asymmetric distribution. Clearly, it would be interesting to go more deeply into this comparison and in this work we present for the first time simulation studies that examine the maximum asymptotic bias, which is considered to be the most accurate measure of robustness for point estimators.

The rest of the paper is structured as follows. Preliminaries on the space of fuzzy numbers are recalled in Section 2. Section 3 reviews location estimators of fuzzy number-valued data. Section 4 introduces the maximum asymptotic bias for fuzzy number-valued estimators and Section 5 contains the empirical comparison of the finite-sample behaviour of fuzzy trimmed means, Huber and Hampel fuzzy M-estimates, the 1-norm median and the wabl/ldev/rdev median with respect to this important measure of robustness. Finally, some concluding remarks are provided in Section 6. 


\section{The space of fuzzy numbers}

A (bounded) fuzzy number is a mapping $\widetilde{U}: \mathbb{R} \rightarrow[0,1]$ such that its $\alpha$-levels

$$
\widetilde{U}_{\alpha}= \begin{cases}\{x \in \mathbb{R}: \widetilde{U}(x) \geq \alpha\} & \text { if } \alpha \in(0,1] \\ \operatorname{cl}\{x \in \mathbb{R}: \widetilde{U}(x)>0\} & \text { if } \alpha=0,\end{cases}
$$

where cl denotes the closure, are nonempty compact intervals. The space of all fuzzy numbers is denoted by $\mathcal{F}_{c}(\mathbb{R})$. Regarding the interpretation of a fuzzy number, $\widetilde{U}(x)$ represents the 'degree of compatibility' of $x$ with $\widetilde{U}$ ' (or 'degree of truth' of the assertion " $x$ is $\widetilde{U}$ "). Therefore, fuzzy numbers are suitable to describe human valuations, such as ratings, judgments, opinions or perceptions. Due to their gradualness the $\alpha$-levels add to the imprecision of interval-valued data.

One of the ways to characterize a fuzzy number $\widetilde{U}$ in a unique manner is to consider the infima and suprema of all its $\alpha$-levels. Alternatively, another characterization can be obtained by defining the following indicators (see [7] for more details):

- The weighted average based on levels (wabl) as a measure of the "center': it represents the real number in the interior set $\operatorname{int}\left(\widetilde{U}_{0}\right)$ such that

$$
\operatorname{wabl}(\widetilde{U})=\int_{[0,1]} \operatorname{mid} \widetilde{U}_{\alpha} d \ell(\alpha)
$$

with $\ell$ the Lebesgue measure and $\operatorname{mid} \widetilde{U}_{\alpha}=\left(\inf \widetilde{U}_{\alpha}+\sup \widetilde{U}_{\alpha}\right) / 2$.

- The left deviation with respect to wabl

$$
\operatorname{ldev}_{\widetilde{U}}:[0,1] \rightarrow \mathbb{R}, \quad \alpha \mapsto \operatorname{ldev}_{\widetilde{U}}(\alpha)=\operatorname{wabl}(\widetilde{U})-\inf \widetilde{U}_{\alpha}
$$

- The right deviation with respect to wabl

$$
\operatorname{rdev}_{\widetilde{U}}:[0,1] \rightarrow \mathbb{R}, \quad \alpha \mapsto \operatorname{rdev}_{\widetilde{U}}(\alpha)=\sup \widetilde{U}_{\alpha}-\operatorname{wabl}(\widetilde{U})
$$

Zadeh's extension principle provides us with the following operations to handle these data.

Definition 2.1. Let $\widetilde{U}, \widetilde{V} \in \mathcal{F}_{c}(\mathbb{R})$. The sum of $\widetilde{U}$ and $\widetilde{V}$ is defined as the fuzzy number $\widetilde{U}+\widetilde{V} \in \mathcal{F}_{c}(\mathbb{R})$ which for each $\alpha \in[0,1]$ is given by

$$
(\widetilde{U}+\widetilde{V})_{\alpha}=\left[\inf \widetilde{U}_{\alpha}+\inf \widetilde{V}_{\alpha}, \sup \widetilde{U}_{\alpha}+\sup \widetilde{V}_{\alpha}\right]
$$


Let $\widetilde{U} \in \mathcal{F}_{c}(\mathbb{R})$ and $\gamma \in \mathbb{R}$. The product of $\widetilde{U}$ by the scalar $\gamma$ is defined as the fuzzy number $\gamma \cdot \widetilde{U} \in \mathcal{F}_{c}(\mathbb{R})$ which for each $\alpha \in[0,1]$ is given by

$$
(\gamma \cdot \widetilde{U})_{\alpha}= \begin{cases}{\left[\gamma \cdot \inf \widetilde{U}_{\alpha}, \gamma \cdot \sup \widetilde{U}_{\alpha}\right]} & \text { if } \gamma \geq 0 \\ {\left[\gamma \cdot \sup \widetilde{U}_{\alpha}, \gamma \cdot \inf \widetilde{U}_{\alpha}\right]} & \text { otherwise }\end{cases}
$$

Alternatively, the sum and the product by a scalar can be defined in terms of the second characterization since

- $\operatorname{wabl}(\widetilde{U}+\widetilde{V})=\operatorname{wabl}(\widetilde{U})+\operatorname{wabl}(\widetilde{V})$, $\operatorname{ldev}_{\widetilde{U}+\widetilde{V}}(\alpha)=\operatorname{ldev}_{\widetilde{U}}(\alpha)+\operatorname{ldev}_{\widetilde{V}}(\alpha), \operatorname{rdev}_{\widetilde{U}+\widetilde{V}}(\alpha)=\operatorname{rdev}_{\widetilde{U}}(\alpha)+\operatorname{rdev}_{\widetilde{V}}(\alpha)$.

- $\operatorname{wabl}(\gamma \cdot \widetilde{U})=\gamma \cdot \operatorname{wabl}(\widetilde{U})$,

$$
\begin{aligned}
& \operatorname{ldev}_{\gamma \cdot \widetilde{U}}(\alpha)= \begin{cases}\gamma \cdot \operatorname{ldev}_{\widetilde{U}}(\alpha) & \text { if } \gamma \geq 0, \\
-\gamma \cdot \operatorname{rdev}_{\widetilde{U}}(\alpha) & \text { otherwise, }\end{cases} \\
& \operatorname{rdev}_{\gamma \cdot \widetilde{U}}(\alpha)= \begin{cases}\gamma \cdot \operatorname{rdev}_{\widetilde{U}}(\alpha) & \text { if } \gamma \geq 0, \\
-\gamma \cdot \operatorname{ldev}_{\widetilde{U}}(\alpha) & \text { otherwise. }\end{cases}
\end{aligned}
$$

The operations above are indeed a level-wise extension of the usual interval arithmetic. The space $\mathcal{F}_{c}(\mathbb{R})$ endowed with these two operations is not linear because of the lack of an opposite element for the sum. There does not exist an always well-defined concept of difference between fuzzy numbers which keeps the properties of the difference operation in connection with the sum in the real settings. Therefore, for statistical developments differences will be replaced by distances between fuzzy numbers whenever possible. This fact highlights the crucial role metrics play in the statistical analysis of fuzzy number-valued data (for more details, see [10] and [11]). In this work we consider the family of distances between fuzzy numbers introduced by Montenegro et al. [12], which extends the metrics defined by Bertoluzza et al. [13].

Definition 2.2. The mid/spr-based $L^{2}$ distance between any two fuzzy numbers $\widetilde{U}, \widetilde{V} \in \mathcal{F}_{c}(\mathbb{R})$ is defined as

$$
D_{\theta}(\widetilde{U}, \widetilde{V})=\sqrt{\int_{[0,1]}\left(\left(\operatorname{mid} \widetilde{U}_{\alpha}-\operatorname{mid} \widetilde{V}_{\alpha}\right)^{2}+\theta\left(\operatorname{spr} \widetilde{U}_{\alpha}-\operatorname{spr} \widetilde{V}_{\alpha}\right)^{2}\right) d \ell(\alpha)},
$$

where $\theta \in(0,+\infty)$, mid $\widetilde{U}_{\alpha}=\left(\inf \widetilde{U}_{\alpha}+\sup \widetilde{U}_{\alpha}\right) / 2$ as before and $\operatorname{spr} \widetilde{U}_{\alpha}$ $=\left(\sup \widetilde{U}_{\alpha}-\inf \widetilde{U}_{\alpha}\right) / 2$. 
This family of distances between fuzzy numbers is very versatile since the nonnegative parameter $\theta$ allows to weigh the relative importance assigned to the 'deviation in shape' (represented by spr) in contrast to the 'deviation in center' (represented by mid). Notice that $\theta$ does not have any stochastic meaning. Along this work, the common choice $\theta=1 / 3$ is used. The distance $D_{1 / 3}$ is a popular choice because it assigns the same relevance to all the points in the intervals. Indeed, $D_{1 / 3}$ can be alternatively expressed as

$$
D_{1 / 3}(\widetilde{U}, \widetilde{V})=\sqrt{\int_{[0,1]}\left(\widetilde{U}_{\alpha}^{[\eta]}-\widetilde{V}_{\alpha}^{[\eta]}\right)^{2} d \ell(\eta)},
$$

with $\ell=$ Lebesgue measure on $\left([0,1], \mathcal{B}_{[0,1]}\right)$ and $\widetilde{U}_{\alpha}^{[\eta]}=\eta \cdot \sup \widetilde{U}_{\alpha}+(1-\eta)$. $\inf \widetilde{U}_{\alpha}$ for all $\eta \in[0,1]$.

The mid/spr representation is not considered as an alternative to characterize fuzzy numbers because, in contrast to the inf/sup representation or the wabl/ldev/rdev representation, one cannot state a set of necessary and sufficient conditions for the functions involved in the mid/spr representation to characterize a fuzzy number.

In statistics data are assumed to come from the repeated observation of a random mechanism, so we now recall the notion of random fuzzy number in Puri and Ralescu's sense $[14,15]$. This concept determines a well-stated and supported model for the random mechanism generating fuzzy number-valued data within the probabilistic setting. A random fuzzy number integrates both randomness (which affects the generation of experimental data) and imprecision (which reflects the nature of the experimental data).

Definition 2.3. Let $(\Omega, \mathcal{A}, P)$ be a probability space modeling a random experiment. A mapping $\mathcal{X}: \Omega \rightarrow \mathcal{F}_{c}(\mathbb{R})$ is said to be a random fuzzy number associated with the random experiment if and only if, for each $\alpha \in[0,1]$ the interval-valued mapping $\mathcal{X}_{\alpha}$ (where $\mathcal{X}_{\alpha}(\omega)=(\mathcal{X}(\omega))_{\alpha}$ for all $\omega \in \Omega$ ) is a random compact interval or, equivalently, the real-valued functions inf $\mathcal{X}_{\alpha}$ and $\sup \mathcal{X}_{\alpha}$ are random variables.

Notice that notions like the induced distribution of a random fuzzy number or the stochastic independence of random fuzzy numbers do not need to be stated explicitly, due to the Borel-measurability of random fuzzy numbers with respect to the Borel $\sigma$-field associated with the mid/spr-based $L^{2}$ distance, among other metrics (see e.g. [16]). 


\section{Location estimators of fuzzy number-valued data}

In this section, different measures that can be found in the literature to summarize the central tendency of a sample of fuzzy number-valued observations are recalled. Let $\widetilde{\mathbf{x}}_{n}=\left(\widetilde{x}_{1}, \ldots, \widetilde{x}_{n}\right)$ be a sample of fuzzy number-valued observations from a random fuzzy number $\mathcal{X}$.

Definition 3.1. The sample Aumann-type mean [2] is the fuzzy number $\overline{\widetilde{\mathbf{x}}}_{n}$ such that for all $\alpha \in[0,1]$ its $\alpha$-levels are given by

$$
\left(\overline{\widetilde{\mathbf{x}}}_{n}\right)_{\alpha}=\left[\sum_{i=1}^{n} \inf \left(\widetilde{x}_{i}\right)_{\alpha} / n, \sum_{i=1}^{n} \sup \left(\widetilde{x}_{i}\right)_{\alpha} / n\right] .
$$

As already mentioned in Section 1, the Aumann-type mean is heavily influenced by outliers and data changes. This has motivated the search for more robust location measures and, in this way, some successful alternatives from the classical settings have been adapted to the fuzzy number-valued case. We start with the extensions of trimmed means and M-estimators of location.

Definition 3.2. Let $\theta \in(0,+\infty)$. For any $\beta \in(0,1)$, the sample fuzzy trimmed mean [6] is the fuzzy number $\frac{1}{h} \sum_{j \in \widehat{E}_{\widetilde{\mathbf{x}}_{\mathbf{n}}}} \tilde{x}_{j}$, where $\widehat{E}_{\widetilde{\mathbf{x}}_{\mathbf{n}}}$ denotes the corresponding sample trimming region, that is,

$$
\begin{gathered}
\widehat{E}_{\widetilde{\mathbf{x}}_{\mathbf{n}}}=\arg \min _{\substack{E \subset\{1, \ldots, n\} \\
\# E=h}} \frac{1}{h} \sum_{i \in E}\left(D_{\theta}\left(\tilde{x}_{i}, \frac{1}{h} \sum_{j \in E} \tilde{x}_{j}\right)\right)^{2} \\
=\arg \min _{E \in \mathcal{E}} \operatorname{Var}\left(\widetilde{\mathbf{x}}_{\mathbf{n}} \mid E\right)
\end{gathered}
$$

with the set $\mathcal{E}=\{E \subset\{1, \ldots, n\}: \# E=h\}$ consisting of all the subsets of $h=n-\lfloor n \beta\rfloor$ different natural numbers which are up to the sample size.

Definition 3.3. The sample fuzzy M-estimator of location associated with a certain loss function $\rho$ [4] is the fuzzy number that minimizes the expression $\frac{1}{n} \sum_{i=1}^{n} \rho\left(D_{\theta}\left(\widetilde{x}_{i}, \widetilde{U}\right)\right)$, over $\widetilde{U} \in \mathcal{F}_{c}(\mathbb{R})$ (if it exists).

For the empirical study in Section 4, we have chosen the Huber and Hampel families of loss functions in order to complement the simulations presented in [9]. The Huber loss function is given by

$$
\rho_{a}^{H}(x)= \begin{cases}x^{2} / 2 & \text { if } 0 \leq x \leq a \\ a(x-a / 2) & \text { otherwise }\end{cases}
$$


with $a>0$ a tuning parameter, and the Hampel loss function is given by

$$
\rho_{a, b, c}(x)= \begin{cases}x^{2} / 2 & \text { if } 0 \leq x<a \\ a(x-a / 2) & \text { if } a \leq x<b \\ \frac{a(x-c)^{2}}{2(b-c)}+\frac{a(b+c-a)}{2} & \text { if } b \leq x<c \\ \frac{a(b+c-a)}{2} & \text { if } c \leq x,\end{cases}
$$

where the nonnegative parameters $a<b<c$ control the degree of suppression of large errors. The smaller their values, the higher this degree.

Whereas the Huber loss function is convex, Hampel's family of loss functions is not convex anymore. The Huber loss function puts less emphasis on large errors than the squared error loss and thus yields a more robust location estimator than the Aumann-type mean. Moreover, in the Hampel loss function all large errors (larger than $c$ ) contribute in the same way to the loss, so it can cope even better with extreme outliers.

The following two measures are also particular cases of fuzzy M-estimators of location. In both cases the loss function $\rho$ is the absolute value function, but for different metrics. For that reason, they are extensions of the concept of median of a real-valued random variable.

Definition 3.4. The sample 1-norm median [8] is the fuzzy number such that for all $\alpha \in[0,1]$ the corresponding $\alpha$-level is given by the interval

$$
\left[\operatorname{Me}\left(\left\{\inf \left(\widetilde{x}_{i}\right)_{\alpha}\right\}_{i=1}^{n}\right), \operatorname{Me}\left(\left\{\sup \left(\widetilde{x}_{i}\right)_{\alpha}\right\}_{i=1}^{n}\right)\right] .
$$

Definition 3.5. The sample wabl/ldev/rdev-median [5] is the fuzzy number such that for all $\alpha \in[0,1]$ the corresponding $\alpha$-level is given by the interval

$$
\begin{aligned}
& {\left[\operatorname{Me}\left(\left\{\operatorname{wabl}\left(\widetilde{x}_{i}\right)\right\}_{i=1}^{n}\right)-\operatorname{Me}\left(\left\{\operatorname{ldev}\left(\widetilde{x}_{i}\right)_{\alpha}\right\}_{i=1}^{n}\right),\right.} \\
& \left.\operatorname{Me}\left(\left\{\operatorname{wabl}\left(\widetilde{x}_{i}\right)\right\}_{i=1}^{n}\right)+\operatorname{Me}\left(\left\{\operatorname{rdev}\left(\widetilde{x}_{i}\right)_{\alpha}\right\}_{i=1}^{n}\right)\right] .
\end{aligned}
$$

The robustness of these measures has been previously studied in terms of the finite sample breakdown point, which is the minimum proportion of sample data that has to be modified in order to make the distance between the M-estimates of the original data and the contaminated data arbitrarily large. 
The sample 1-norm median and the sample wabl/ldev/rdev-median always attain the maximum finite sample breakdown point, $\frac{1}{n}\left\lfloor\frac{n+1}{2}\right\rfloor$. Under general conditions (see [4]), Huber and Hampel fuzzy M-estimators also obtain the maximum finite sample breakdown point, whereas fuzzy trimmed means will only reach the maximal value $\frac{1}{n}\left\lfloor\frac{n+1}{2}\right\rfloor$ for trimming proportion $\beta=0.5$.

In [9], the bias, the variance and the mean squared error of all these location measures (also including the Aumann-type mean) were computed with the aim of comparing the finite-sample behaviour of the estimators. No uniformly most appropriate location estimate could be chosen, as in the classical settings, but it could be concluded that Huber and Hampel fuzzy M-estimators in general behave well for small contamination levels while fuzzy trimmed means performed better for larger contamination proportions. However, it must be clarified that such results depend on the chosen distributions and in some cases the distinction between fuzzy M-estimators and fuzzy trimmed means is not as evident. Therefore, it would be really interesting to complement these comparative studies with other situations and also the computation of alternative measures of robustness. To this end, the next section is focused on the introduction of the maximum asymptotic bias, a new measure of robustness for fuzzy number-valued estimators.

\section{Maximum asymptotic bias}

The finite sample breakdown point analyzed in previous studies measures the largest proportion of contamination that an estimator can tolerate and thus is a measure of robustness that focuses on extreme situations. Consider a real-valued estimator $\widehat{T}$ and let $\widehat{T}_{n}=\widehat{T}_{n}\left(\mathbf{x}_{n}\right)$ denote the estimate for a sample $\mathbf{x}_{n}=\left\{x_{1}, \ldots, x_{n}\right\}$ which is a realization of $n$ i.i.d. real-valued random variables with distribution $F$. The finite sample breakdown point of $\widehat{T}_{n}$, denoted by $\varepsilon_{n}^{*}\left(\widehat{T}_{n}, \mathbf{x}_{n}\right)$, usually does not depend on the random sample $\mathbf{x}_{n}$, and tends to the asymptotic breakdown point of $\widehat{T}$, denoted by $\varepsilon^{*}(\widehat{T}, F)$ :

$$
\varepsilon_{n}^{*}\left(\widehat{T}_{n}, \mathbf{x}_{n}\right) \underset{n \rightarrow \infty}{\longrightarrow} \varepsilon^{*}(\widehat{T}, F) .
$$

Now, let us consider the asymptotic value of the estimator, $\widehat{T}_{\infty}(F)$, defined as the limit in probability of the estimator $\widehat{T}_{n}\left(\mathbf{x}_{n}\right)$ :

$$
\widehat{T}_{n} \stackrel{P}{\rightarrow} \widehat{T}_{\infty}(F) .
$$


This asymptotic value of the estimator at $F$ exists in all cases of practical interest. As Maronna et al. [17] explain, a high breakdown point for an estimator is associated with an asymptotic value $\widehat{T}_{\infty}(F)$ which more often belongs to a bounded set. In detail, consider an $\varepsilon$-neighborhood $\mathcal{F}_{\varepsilon, \vartheta}$ of an assumed parametric distribution $F_{\vartheta}$, which is defined by

$$
\mathcal{F}_{\varepsilon, \vartheta}=\left\{(1-\varepsilon) F_{\vartheta}+\varepsilon G: G \in \mathcal{G}\right\},
$$

where $\mathcal{G}$ is a family of distribution functions. If the estimator $\widehat{T}$ has asymptotic breakdown point $\varepsilon^{*}\left(\widehat{T}, F_{\vartheta}\right)$, then this means that $\widehat{T}_{\infty}(F)$ is contained in a bounded set whenever the distribution $F$ ranges in $\mathcal{F}_{\varepsilon, \vartheta}$ with $\varepsilon \leq \varepsilon^{*}\left(\widehat{T}, F_{\vartheta}\right)$. However, the bounded set containing $\widehat{T}_{\infty}(F)$ may be very large. For that reason, a new robustness measure arises in order to analyze how the estimator behaves exactly when $\varepsilon<\varepsilon^{*}\left(\widehat{T}, F_{\vartheta}\right)$.

The asymptotic bias of an estimator $\widehat{T}$ at any distribution $F \in \mathcal{F}_{\varepsilon, \vartheta}$ is defined as

$$
b_{\widehat{T}}(F, \vartheta)=\widehat{T}_{\infty}(F)-\widehat{T}_{\infty}\left(F_{\vartheta}\right),
$$

and the maximum asymptotic bias (or maxbias for short) of $\widehat{T}$ at $F_{\vartheta}$ is given by

$$
M B_{\widehat{T}}(\varepsilon, \vartheta)=\max \left\{\left|b_{\widehat{T}}(F, \vartheta)\right|: F \in \mathcal{F}_{\varepsilon, \vartheta}\right\} .
$$

The maximum asymptotic bias, originally defined by Huber [18, 19], is considered the most powerful tool to compare the robustness of estimators since, given two estimators, the one with smaller maximum asymptotic bias curve is clearly more robust [20].

\subsection{Maximum asymptotic bias of fuzzy number-valued estimators}

In this section, the notion of maximum asymptotic bias is adapted to cover the fuzzy number-valued case.

Definition 4.1. Given a metric $D$ defined on $\mathcal{F}_{c}(\mathbb{R}) \times \mathcal{F}_{c}(\mathbb{R})$, the asymptotic D-bias of a fuzzy number-valued estimator $\widehat{\widetilde{T}}$ at any distribution $F \in \mathcal{F}_{\varepsilon, \vartheta}$ on $\mathcal{F}_{c}(\mathbb{R})$ is

$$
b_{\widetilde{\widetilde{T}}}(F, \vartheta)=D\left(\widehat{\widetilde{T}}_{\infty}(F), \widehat{\widetilde{T}}_{\infty}\left(F_{\vartheta}\right)\right)
$$

and the maximum asymptotic D-bias (or maxbias for short) of $\widehat{\widetilde{T}}$ at $F_{\vartheta}$ is

$$
M B_{\widehat{\widetilde{T}}}(\varepsilon, \vartheta)=\max _{F \in \mathcal{F}_{\varepsilon, \vartheta}} D\left(\widehat{\widetilde{T}}_{\infty}(F), \widehat{\widetilde{T}}_{\infty}\left(F_{\vartheta}\right)\right)
$$


Notice that, as commented in Section 2, the difference between real numbers in (1) which yields the asymptotic bias of a real-valued estimator has been replaced by a distance between fuzzy numbers in Definition 4.1.

Under general conditions, the location measures considered in this work are all strongly consistent with respect to the metric involved in their definition (the strong consistency of fuzzy trimmed means derives from the study of the general Hilbert-valued case by Cuesta-Albertos and Fraiman [21, 22]; for fuzzy M-estimators, the Aumann-type mean, the 1-norm median and the wabl/ldev/rdev median see [4], [23], [8] and [5], respectively). Fuzzy trimmed means, fuzzy M-estimators and the Aumann-type mean are defined in terms of the $D_{\theta}$-metric, whereas the 1-norm median is based on the 1-norm distance $[24]$ between two fuzzy numbers $\widetilde{U}$ and $\widetilde{V}$, given by

$$
\rho_{1}(\widetilde{U}, \widetilde{V})=\frac{1}{2} \int_{[0,1]}\left(\left|\inf \widetilde{U}_{\alpha}-\inf \widetilde{V}_{\alpha}\right|+\left|\sup \widetilde{U}_{\alpha}-\sup \widetilde{V}_{\alpha}\right|\right) d \ell(\alpha)
$$

and the wabl/ldev/rdev median involves the wabl/ldev/rdev-based $L^{1}$ metric [7], which is defined as

$$
\begin{gathered}
\mathcal{D}_{\theta}(\widetilde{U}, \widetilde{V})=|\operatorname{wabl}(\widetilde{U})-\operatorname{wabl}(\widetilde{V})| \\
+\frac{\theta}{2} \int_{[0,1]}\left|\operatorname{ldev}_{\widetilde{U}}(\alpha)-\operatorname{ldev}_{\widetilde{V}}(\alpha)\right| d \ell(\alpha)+\frac{\theta}{2} \int_{[0,1]}\left|\operatorname{rdev}_{\widetilde{U}}(\alpha)-\operatorname{rdev}_{\widetilde{V}}(\alpha)\right| d \ell(\alpha) .
\end{gathered}
$$

Note that the metrics mentioned above are topologically equivalent. The strong consistency of the location measures (with respect to any of these metrics due to the topological equivalence) implies that the asymptotic value of these estimators coincide with their population location measure. Hence, if $\widetilde{T}_{F}$ represents the population location value of an estimator $\widehat{\widetilde{T}}$ at a distribution $F$ and the general conditions to guarantee the strong consistency of $\widehat{\widetilde{T}}$ hold, $\widetilde{T}_{F}=\widehat{\widetilde{T}}_{\infty}(F)$ and

$$
\begin{gathered}
b_{\widetilde{T}}(F, \vartheta)=D\left(\widetilde{T}_{F}, \widetilde{T}_{F_{\vartheta}}\right), \\
M B_{\widetilde{\widetilde{T}}}(\varepsilon, \vartheta)=\max _{F \in \mathcal{F}_{\varepsilon, \vartheta}} D\left(\widetilde{T}_{F}, \widetilde{T}_{F_{\vartheta}}\right) .
\end{gathered}
$$

\section{Empirical comparison of fuzzy number-valued estimators}

The aim of this section is to empirically compute the maximum asymptotic $D$-bias of all the fuzzy number-valued location measures defined in 
Section 3. This will give us more insights into the robustness of the estimators and allow us to make a more detailed comparison of their robustness at various contamination levels. The conducted simulation study consists of two parts:

1. In the first part, maxbias curves are plotted for the fuzzy number-valued location measures.

2. In the second part, for a fixed $\varepsilon$, the asymptotic bias of the fuzzy number-valued location measures is plotted as a function of the outlier location or shape.

\subsection{Empirical study of the maxbias curve}

First, the maxbias curve is plotted for all the considered fuzzy numbervalued location measures. The maxbias curve of an estimator $\widehat{\widetilde{T}}$ at a fixed $F_{\vartheta}$ is a plot of the maximum asymptotic bias $M B_{\widehat{\widetilde{T}}}(\varepsilon, \vartheta)$ as a function of $\varepsilon$. Thus, the maxbias curve informs about the maximal asymptotic bias that an estimator can present when a fraction $\varepsilon$ of the data is contaminated. Note that the curve is obtained by letting $\varepsilon$ vary between zero and its asymptotic breakdown value $\varepsilon^{*}\left(\widehat{\widetilde{T}}, F_{\vartheta}\right)$, which is the highest fraction of contamination that can be withstood by the estimator as mentioned before.

Unfortunately, calculating the maxbias exactly is a very difficult problem that has only been solved for a limited number of estimators in the real setting. Therefore, one usually resorts to an empirically computed approximation (lower bound) for the maxbias of an estimator $\widehat{\widetilde{T}}$. Here, the maxbias curves of the location measures have been empirically approximated by following the next simulation steps:

Step 1. For each of the different situations, 1000 (non-contaminated) samples of $N=1000$ trapezoidal fuzzy number-valued data have been simulated from a random fuzzy number $\mathcal{X}$. We have used four real-valued random variables $X_{1} \sim \mathcal{N}(0,1)$ and $X_{2}, X_{3}, X_{4} \sim \chi_{1}^{2}$ to generate the trapezoidal fuzzy data $\mathcal{X}=\operatorname{Tra}\left(X_{1}-X_{2}-X_{3}, X_{1}-X_{2}, X_{1}+X_{2}, X_{1}+\right.$ $\left.X_{2}+X_{4}\right)$ where $X_{1}=\operatorname{mid} \mathcal{X}_{1}, X_{2}=\operatorname{spr} \mathcal{X}_{1}, X_{3}=\inf \mathcal{X}_{1}-\inf \mathcal{X}_{0}$ and $X_{4}=\sup \mathcal{X}_{0}-\sup \mathcal{X}_{1}$.

Step 2. The population location measures (Aumann-type mean, fuzzy trimmed means with $\beta=0.1$ and $\beta=0.5$, Huber and Hampel fuzzy Mestimators of location, 1-norm median and wabl/ldev/rdev median) 
have been approximated by Monte Carlo simulation using the samples from Step 1.

Step 3. A fraction $\varepsilon$ of contamination (also denoted by $c_{p}$ in analogy to previous simulation studies concerning robust location estimators of fuzzy number-valued data) has been introduced in the fuzzy number-valued data. We have distinguished four different scenarios for the generation of the contaminated data:

Scenario 3.1 Contaminated data are real-valued and we study the effect of point contamination at any point $C_{D}$, where $C_{D}$ ranges in $\{0,100,200,300,400,500\}$.

Scenario 3.2 Contaminated data are the fuzzy numbers $\operatorname{Tra}\left(C_{D}-2, C_{D}-\right.$ $\left.1, C_{D}+1, C_{D}+2\right)$, which represents a translation of the population Aumann-type mean, $\operatorname{Tra}(-2,-1,1,2)$, with $C_{D}$ units (therefore, contaminated data have the same shape as the Aumann-type mean).

Scenario 3.3 Contaminated data are the fuzzy numbers $\operatorname{Tra}\left(-2 C_{D},-C_{D}\right.$, $\left.C_{D}, 2 C_{D}\right)$ that are symmetric with respect to 0 and represent the product of $C_{D}$ times the population Aumann-type mean.

Scenario 3.4 Contaminated data are the fuzzy numbers $\operatorname{Tra}\left(Y_{1}-Y_{2}-\right.$ $\left.Y_{3}, Y_{1}-Y_{2}, Y_{1}+Y_{2}, Y_{1}+Y_{2}+Y_{4}\right)$ generated from the real-valued random variables $Y_{1} \sim \mathcal{N}\left(C_{D}, 3\right)$ and $Y_{2}, Y_{3}, Y_{4} \sim \chi_{4}^{2}+C_{D}$.

Step 4. For each scenario of Step 3 and each value of $C_{D}$, the population location measures (Aumann-type mean, fuzzy trimmed means with $\beta=0.1$ and $\beta=0.5$, Huber and Hampel fuzzy M-estimators of location, 1-norm median and wabl/ldev/rdev median) have been approximated by Monte Carlo simulation using the contaminated samples from Step 3.

Step 5. For each scenario of Step 3 and each value of $C_{D}$, the $\rho_{1}$ and $D_{\theta}$ distances, $\theta \in\{1 / 3,1\}$, between the non-contaminated and the contaminated approximate population measures have been computed. Then, the maximum $\rho_{1}$ and $D_{\theta}$ distances, $\theta \in\{1 / 3,1\}$, for each location measure have been computed over the four scenarios and all values of $C_{D}$. 
Step 6. The interval $[0,0.49]$ has been partitioned in 10 equidistant parts and for each of the 11 equidistant points Steps 1-5 have been repeated. Finally, the maximum $\rho_{1}$ and $D_{\theta}$ distances from Step 5 (i.e., maximum asymptotic biases) have been plotted versus the corresponding value of $\varepsilon$ in Figure 1 .

Please note that only trapezoidal fuzzy numbers have been considered in order to ease the computation, since a sensitivity analysis has shown that the shape of the fuzzy numbers seems to scarcely affect statistical conclusions (see [25] for more details).

Both Huber and Hampel fuzzy M-estimators of location have been computed using two alternative choices for the tuning parameters involved in these loss functions. On the one hand, the common values 1.345 for Huber's loss function and 2, 4 and 8 for Hampel's loss function were considered (see the plots in the left column of Figure 1). On the other hand, Kim and Scott's suggestion of choosing the values of the tuning parameters based on the information of the data set [26] was considered as well (see the plots in the right column of Figure 1). The latter option shows more flexibility since the selected tuning parameters are the median (Huber and Hampel), the $75^{\text {th }}$ percentile and the $85^{\text {th }}$ percentile of the distances between each observation and a robust seed (in these simulation studies, the 1-norm median has been considered as the robust seed). These adaptive choices allow that the tuning constants are adjusted in each iteration to better cope with the new data set and avoid problems due to the lack of scale equivariance of these estimators (for more details about the scale equivariance, see [4]). However, this flexibility results in a larger maximum asymptotic bias when the contamination proportion increases.

The first conclusion that we can draw from Figure 1 is that the maxbias curves of the Aumann-type mean and the fuzzy trimmed mean with $\beta=0.1$ completely differ from the remaining ones. Naturally, this is due to the fact that the Aumann-type mean is always non robust (its asymptotic breakdown point is zero) and the fuzzy trimmed mean with $\beta=0.1$ can only withstand a contamination proportion up to $\varepsilon=0.1$, which means that a larger $\varepsilon$ will make the estimator break down. Notice that the maxbias curve reveals the breakdown point as the value $\varepsilon$ that makes the maximum asymptotic bias arbitrarily large. Of course, the situation is completely different if the trimming proportion $\beta$ is large, as shown for $\beta=0.5$.

The other location measures (the 1 -norm and the wabl/ldev/rdev medi- 

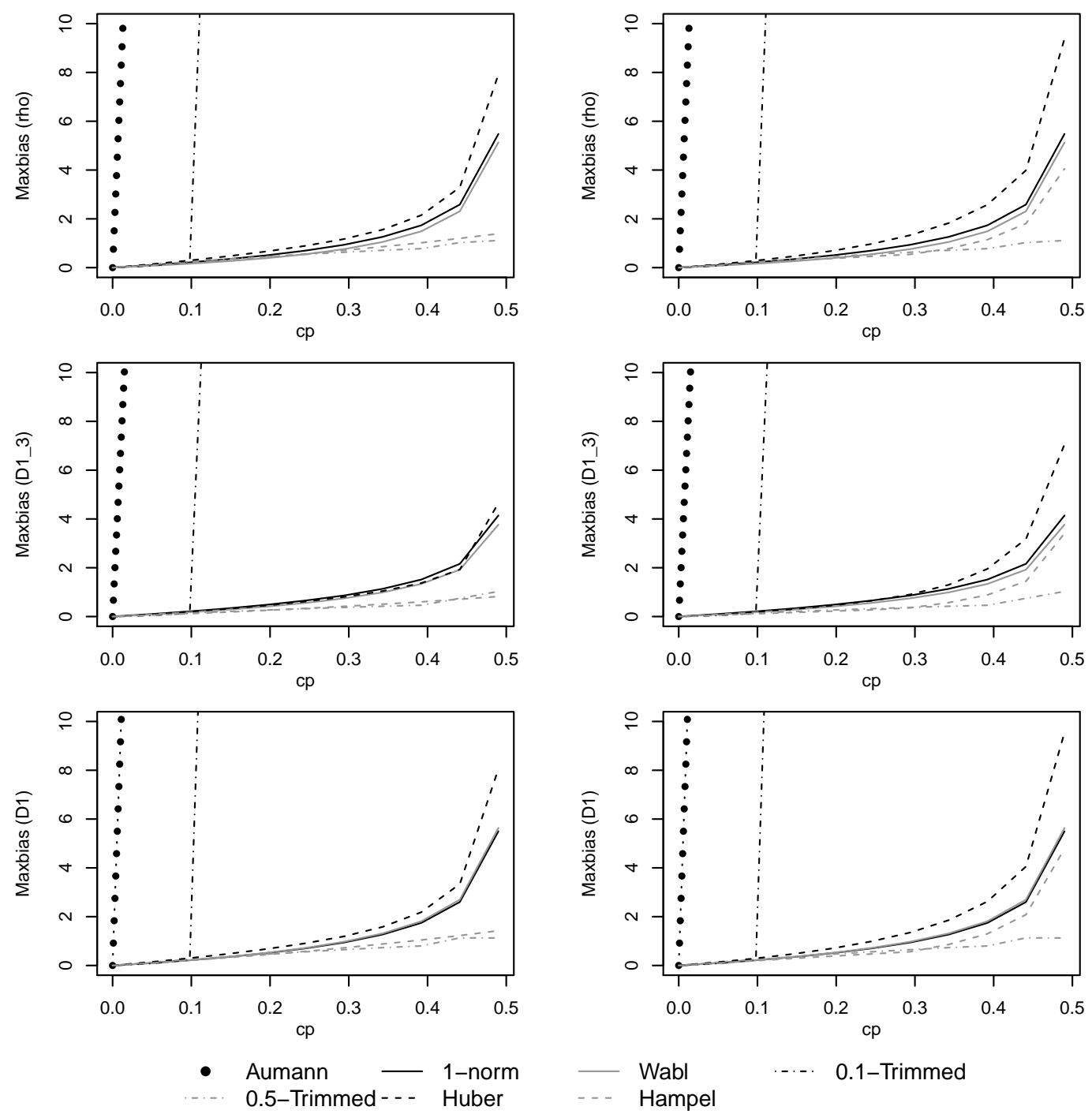

Figure 1: Maximum asymptotic biases of the fuzzy number-valued location measures as a function of $\varepsilon$. The metric used in the computation is $\rho_{1}$ (first row), $D_{1 / 3}$ (second row) or $D_{1}$ (third row). For each situation, the fuzzy M-estimators of location have been computed using a (fixed) common choice for the tunning parameters (plots on the left) or following Kim and Scott's suggestion (plots on the right).

ans, the fuzzy M-estimators and the trimmed mean with $\beta=0.5)$ can tolerate up to $50 \%$ of contamination, so Figure 1 shows their maximum asymptotic bias before their breakdown point $\varepsilon^{*}=0.5$. From their maxbias curves we can now see differences in their behaviour for smaller levels of contamination, but close to 0.5 (for low contamination proportion $c_{p}$, all these maxbias curves are very similar). The maxbias curves of the two concepts of me- 
dian, the 1-norm and the wabl/ldev/rdev median, are very similar (indeed, they are practically indistinguishable when the $D_{1}$ metric is considered). It can be seen that the behaviour of these medians in terms of the maximum asymptotic bias is better than the behaviour of the Huber fuzzy M-estimator of location, but worse than the behaviour of the Hampel fuzzy M-estimator of location. These conclusions remain valid independently from the values of the tuning parameters, but it can also be seen that the maxbias curve of the medians is much closer to the Hampel fuzzy M-estimator's maxbias curve, when Kim and Scott's procedure for chosing the tuning parameters is used. As expected, the adaptive choice of the tuning parameters increases the bias of the Huber and Hampel fuzzy M-estimators for larger fractions of contamination. The best maxbias curve from the study corresponds to the trimmed mean with $\beta=0.5$. It can be noticed that the Hampel fuzzy Mestimator's maxbias curve is very similar to that of the trimmed mean with $\beta=0.5$ when the (fixed) common choice for the tuning parameters is used, but the differences between them are larger when Kim and Scott's procedure is chosen instead.

To evaluate whether the maxbias of an estimator is large or small, we compute the empirical distribution of the distances of non-contaminated data with respect to each of the population location measures as follows. First, the population (non-contaminated) location measures have been approximated by Monte Carlo as in Step 2. Secondly, a sample of size $10^{6}$ has been generated as in Step 1. For each location measure, the $D_{1 / 3}$ distances between each observation and the Monte Carlo approximation of the population measure have been computed and plotted in Figure 2.

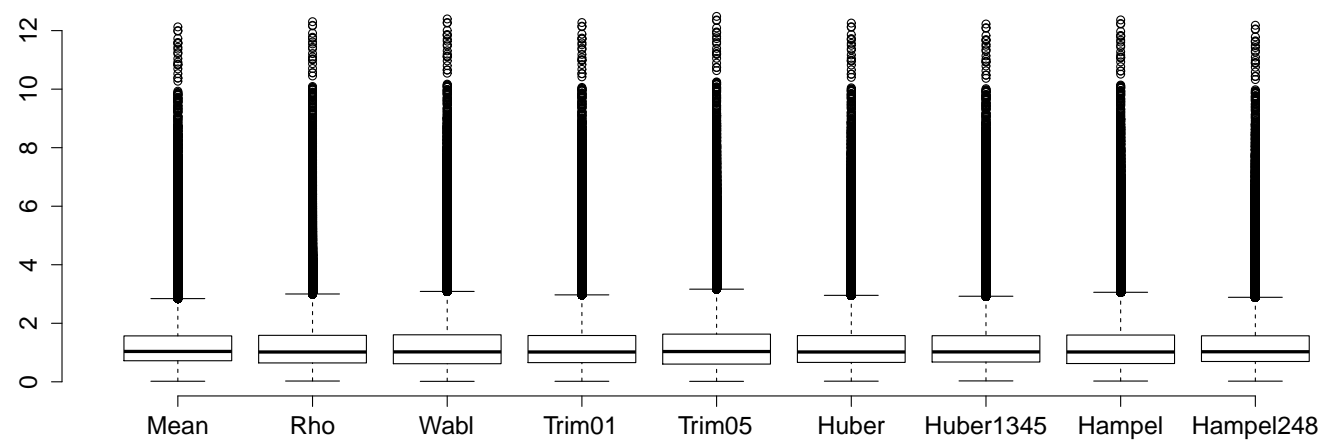

Figure 2: Boxplots of the empirical distribution of $D_{1 / 3}$ distances between the noncontaminated data and the fuzzy number-valued location measures. 
These empirical distributions allow us to evaluate whether a particular value for the bias is small or large. Indeed, from Figure 2 we can conclude that a maxbias above 3.5 can be considered to be large in the sense that the contaminated location estimate lies in the far right tail of the distribution of the distances for the non-contaminated observations. From Figure 1, we can see that only the trimmed mean with $\beta=0.5$ and the Hampel fuzzy M-estimator have maxbiases that remain sufficiently small, even for large percentages of contamination.

\subsection{Empirical study of the asymptotic bias}

Next to the maxbias curves we also examine the asymptotic bias of the location measures, because it could be the case that an estimator with a higher maximum asymptotic bias obtains a much lower asymptotic bias for the majority of the cases. To generate these plots, the following simulation steps have been used:

Step 1. For each of the different situations, 1000 (non-contaminated) samples of $N=1000$ trapezoidal fuzzy number-valued data have been simulated from a random fuzzy number $\mathcal{X}$. We have used four real-valued random variables $X_{1} \sim \mathcal{N}(0,1)$ and $X_{2}, X_{3}, X_{4} \sim \chi_{1}^{2}$ to generate the trapezoidal fuzzy data $\mathcal{X}=\operatorname{Tra}\left(X_{1}-X_{2}-X_{3}, X_{1}-X_{2}, X_{1}+X_{2}, X_{1}+\right.$ $\left.X_{2}+X_{4}\right)$ where $X_{1}=\operatorname{mid} \mathcal{X}_{1}, X_{2}=\operatorname{spr} \mathcal{X}_{1}, X_{3}=\inf \mathcal{X}_{1}-\inf \mathcal{X}_{0}$ and $X_{4}=\sup \mathcal{X}_{0}-\sup \mathcal{X}_{1}$.

Step 2. The population location measures (Aumann-type mean, fuzzy trimmed means with $\beta=0.1$ and $\beta=0.5$, Huber and Hampel fuzzy Mestimators of location, 1-norm median and wabl/ldev/rdev median) have been approximated by Monte Carlo simulation using the samples from Step 1.

Step 3. A fraction of contamination $\varepsilon=0.1$ has been considered. Notice that these figures are an adaptation of those in Maronna et al. [17], which show the biases for point contamination (also with $\varepsilon=0.1$ ) as a function of the outlier location $K$. Taking into account that a fuzzy number-valued datum can be classified as an outlier regarding its location or its shape, we have distinguished three different scenarios for the generation of the contaminated data: 
- Outliers in both location and shape: Contaminated data are realvalued and we study the point contamination at any point $C_{D}$, where $C_{D}$ ranges in a partition of 50 equidistant points in the interval $[0,10]$.

- Outliers in location: Contaminated data are the fuzzy numbers $\operatorname{Tra}\left(C_{D}-2, C_{D}-1, C_{D}+1, C_{D}+2\right)$, which represents a translation of the population Aumann-type mean, $\operatorname{Tra}(-2,-1,1,2)$, with $C_{D}$ units (therefore, contaminated data have the same shape as the Aumann-type mean).

- Outliers in shape: Contaminated data are the fuzzy numbers $\operatorname{Tra}\left(-2 C_{D},-C_{D}, C_{D}, 2 C_{D}\right)$ that are symmetric with respect to 0 and represent the product of $C_{D}$ times the population Aumanntype mean.

Step 4. For each scenario of Step 3 and each value of $C_{D}$, the population location measures (Aumann-type mean, fuzzy trimmed means with $\beta=0.1$ and $\beta=0.5$, Huber and Hampel fuzzy M-estimators of location, 1-norm median and wabl/ldev/rdev median) have been approximated by Monte Carlo simulation using the contaminated samples from Step 3.

Step 5. For each scenario of Step 3 and each value of $C_{D}$, the $\rho_{1}$ and $D_{\theta}$ distances, $\theta \in\{1 / 3,1\}$, between the non-contaminated and the contaminated approximate population measures have been computed.

Figures 3-5 show the resulting distances (i.e., asymptotic biases) versus $C_{D}$. As in Section 5.1, the two options regarding the selection of the tuning parameters have been considered for the computation of the fuzzy M-estimators of location.

Figures 3-5 confirm that the asymptotic bias behaviour of fuzzy M-estimators may be very different depending on the choice of the involved tuning parameters. This effect is most clear for the Hampel fuzzy M-estimator in this simulation study. When the tuning parameters are selected following Kim and Scott's procedure, the maximum asymptotic bias increases a bit, as we commented in Section 5.1, due to the impact of contaminated data that are not far from the non contaminated sample (that is, with a small value of $C_{D}$ ) on the asymptotic bias. However, for intermediate values of $C_{D}$, the asymptotic bias of the estimator is much lower with adaptive tuning parameters 

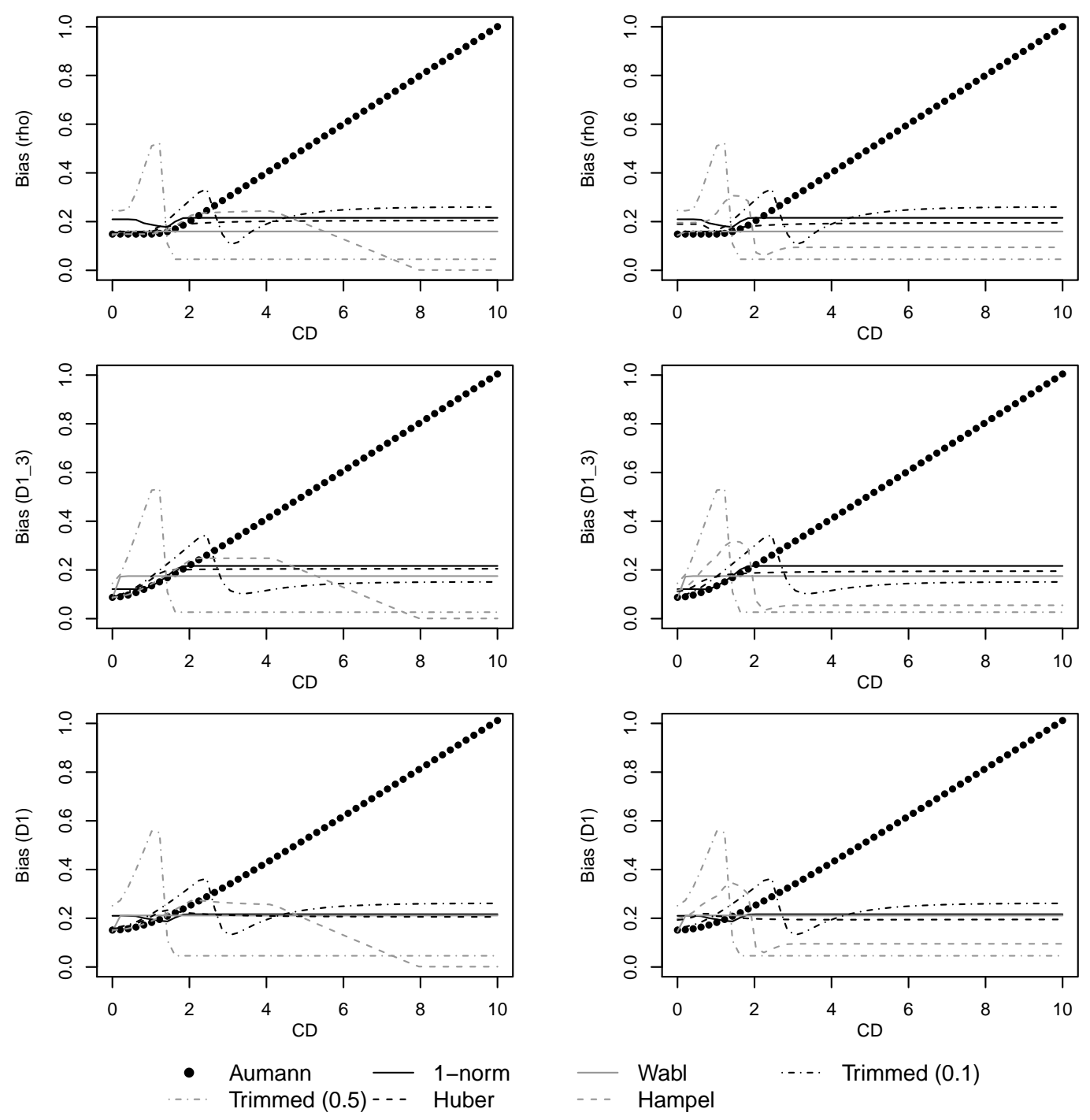

Figure 3: Asymptotic biases of the fuzzy number-valued location measures as a function of $C_{D}(\varepsilon=0.1)$ when there are outliers in both location and shape. The metric used in the computation is $\rho_{1}$ (first row), $D_{1 / 3}$ (second row) or $D_{1}$ (third row). For each situation, the fuzzy M-estimators of location have been computed using a (fixed) common choice for the tuning parameters (plots on the left) or following Kim and Scott's suggestion (plots on the right).

than with fixed valued of these parameters. For that reason, our recommendation is to use Kim and Scott's procedure to select the tuning parameters, not only because it avoids the problems due to the lack of scale equivariance for these fuzzy M-estimators, but also based on its advantages in terms of asymptotic bias. 

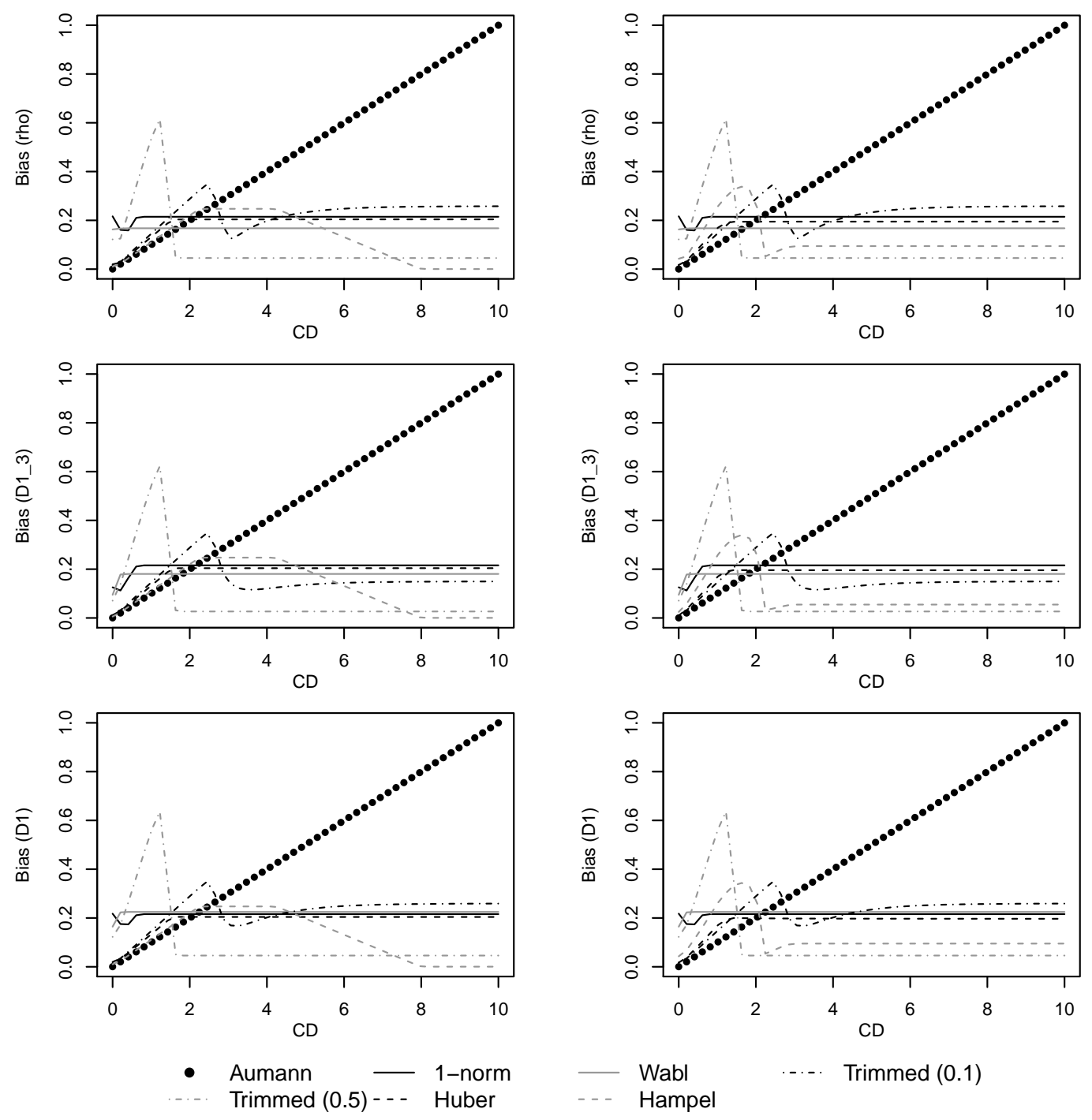

Figure 4: Asymptotic biases of the fuzzy number-valued location measures as a function of $C_{D}(\varepsilon=0.1)$ when there are outliers in location. The metric used in the computation is $\rho_{1}$ (first row), $D_{1 / 3}$ (second row) or $D_{1}$ (third row). For each situation, the fuzzy Mestimators of location have been computed using a (fixed) common choice for the tunning parameters (plots on the left) or following Kim and Scott's suggestion (plots on the right).

It is also interesting to notice that the fuzzy trimmed mean with a trimming proportion suitable for handling the level of contamination in the sample (in this case, $\beta=0.1$ ) may achieve better results than the Huber fuzzy M-estimator. For $C_{D} \geq 2$, the trimmed mean with $\beta=0.5$ yields the best asymptotic bias in general (only improved by the Hampel fuzzy M-estimator for large values of $C_{D}$ when the choice of the tuning parameters is fixed). On 

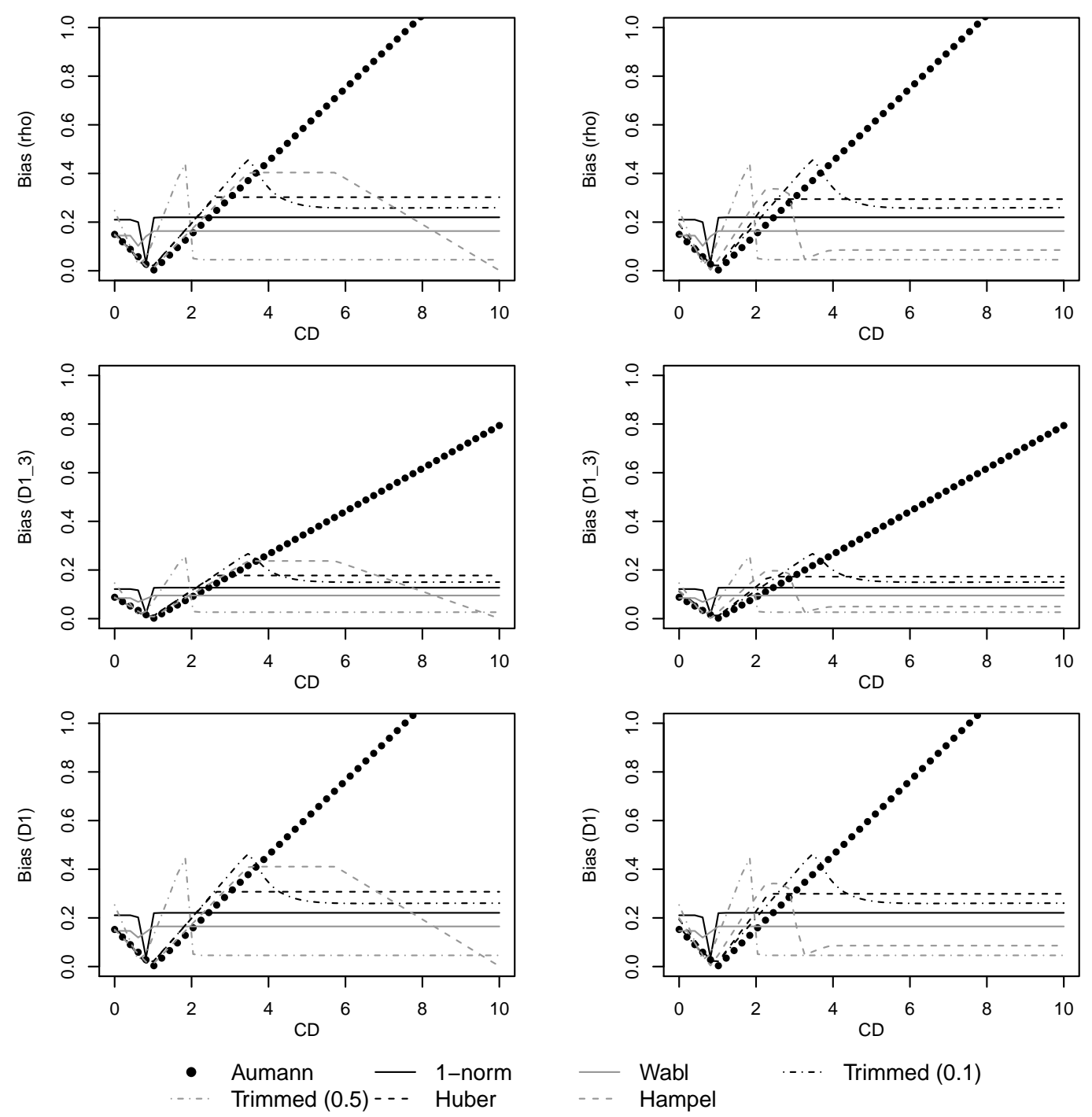

Figure 5: Asymptotic biases of the fuzzy number-valued location measures as a function of $C_{D}(\varepsilon=0.1)$ when there are outliers in shape. The metric used in the computation is $\rho_{1}$ (first row), $D_{1 / 3}$ (second row) or $D_{1}$ (third row). For each situation, the fuzzy Mestimators of location have been computed using a (fixed) common choice for the tunning parameters (plots on the left) or following Kim and Scott's suggestion (plots on the right).

the contrary, for $C_{D}<2$, this trimmed mean is the worst option in terms of the asymptotic bias. Finally, the 1-norm and the wabl/ldev/rdev medians present quite similar asymptotic biases (almost indistinguishable sometimes). Among them, the wabl/ldev/rdev median seems to present the lowest asymptotic bias. Although its behaviour could be improved by the trimmed mean with $\beta=0.5$ and the Hampel fuzzy M-estimator based on Kim and Scott's 
procedure, in general the wabl/ldev/rdev median's asymptotic bias is not higher than the Huber fuzzy M-estimator's asymptotic bias.

The simulation results in this section indicate that the advantages of using the Hampel fuzzy M-estimator of location with tuning parameters selected as in Kim and Scott's procedure in contrast to the use of the 1-norm median or the wabl/ldev/rdev median may be more important than could be assumed when only comparing their maxbias curves. It also happens in the real-valued settings that, "although the maximum bias contains much more information than the breakdown point, it is not informative enough to discriminate among estimators and that one should look at the whole bias behavior when possible", as Maronna et al. [17] stated.

\subsection{Empirical study of the asymptotic variance}

In this section, we complement the studies on the asymptotic bias with the analysis of the relative efficiency of the fuzzy number-valued location measures under non-contamination. Their asymptotic variance has been computed following the next steps:

Step 1. For each of the different situations, the 1000 non-contaminated samples of $N=1000$ trapezoidal fuzzy number-valued data simulated from a random fuzzy number $\mathcal{X}$ in Step 1. of Section 5.2 have been considered. For each of those 1000 samples, the location measures (Aumann-type mean, fuzzy trimmed means with $\beta=0.1$ and $\beta=0.5$, Huber and Hampel fuzzy M-estimators of location, 1-norm median and wabl/ldev/rdev median) have been estimated, $\widehat{T}_{i}$ for $i \in\{1, \ldots, 1000\}$.

Step 2. The Monte Carlo approximation $(T)$ of the corresponding population location measures obtained in Step 2. of Section 5.2 has been considered.

Step 3. The asymptotic variance of each fuzzy number-valued location measure has been approximated by the formula

$$
\operatorname{Var}=\frac{1}{1000} \sum_{i=1}^{1000}\left(D^{2}\left(\widehat{T}_{i}, \frac{1}{1000} \sum_{i=1}^{1000} \widehat{T}_{i}\right)\right)
$$

where $D$ denotes the $\rho_{1}$ and $D_{\theta}$ distances, $\theta \in\{1 / 3,1\}$. 
Table 1 shows the resulting asymptotic variance of each fuzzy numbervalued location measure. As in Sections 5.1 and 5.2, the two options regarding the selection of the tuning parameters have been considered for the computation of the fuzzy M-estimators of location.

Table 1: Asymptotic variances of the fuzzy number-valued location measures computed using the metrics $\rho_{1}, D_{1 / 3}$ and $D_{1}$. The fuzzy M-estimators of location have been computed using a (fixed) common choice for the tunning parameters (see columns Huber and Hampel) or following Kim and Scott's suggestion (see columns Huber* and Hampel*).

\begin{tabular}{cccccc}
\hline Metric & Aumann & 1-norm & Wabl & 0.1 -Trimmed & 0.5-Trimmed \\
\hline$\rho_{1}$ & 0.003047 & 0.0028161 & 0.0029251 & 0.0027199 & 0.0088689 \\
$D_{1 / 3}$ & 0.0021469 & 0.0024992 & 0.00269818 & 0.0023906 & 0.0090213 \\
$D_{1}$ & 0.003731 & 0.0037091 & 0.003818881 & 0.003403 & 0.0099067 \\
\hline Metric & Huber & Huber* & Hampel & Hampel $^{*}$ & \\
\hline$\rho_{1}$ & 0.0022307 & 0.0023068 & 0.0023869 & 0.0031910 & \\
$D_{1 / 3}$ & 0.0018680 & 0.001936 & 0.0018964 & 0.0030530 & \\
$D_{1}$ & 0.002797 & 0.0028918 & 0.0029903 & 0.003873 &
\end{tabular}

The relative efficiency compares the asymptotic variance of two estimators under non-contamination to see which estimator is more precise. In Table 1, the minimum asymptotic variance is achieved by the Huber fuzzy M-estimator of location computed using a (fixed) common choice of the tuning parameter. The behaviour of the Huber fuzzy M-estimator of location based on Kim and Scott's procedure and the Hampel fuzzy M-estimator of location based on a fixed tuning parameter is also very close to the minimum asymptotic variance. However, if we compare the asymptotic variance of any of the remaining estimators with the optimal asymptotic variance, we get larger differences. These relative efficiencies are shown in Table 2.

Therefore, it can be seen that, in general, the fuzzy M-estimators of location provide us with the best asymptotic variances, whilst the asymptotic variance of the fuzzy trimmed mean with $\beta=0.5$ is especially high. 
Table 2: Relative efficiency of each of the fuzzy number-valued location measures and the Huber fuzzy M-estimator of location computed using a (fixed) common choice of the tuning parameter (Huber). The outcomes are shown for the metrics $\rho_{1}, D_{1 / 3}$ and $D_{1}$.

\begin{tabular}{ccccc}
\hline Metric & Aumann & 1-norm & Wabl & 0.1 -Trimmed \\
\hline$\rho_{1}$ & 0.7321709 & 0.7921280 & 0.7625909 & 0.820138 \\
$D_{1 / 3}$ & 0.8700612 & 0.7474401 & 0.6923078 & 0.7813974 \\
$D_{1}$ & 0.7496555 & 0.7541010 & 0.7324161 & 0.8220359 \\
\hline Metric & 0.5 -Trimmed & Huber* & Hampel & Hampel* \\
\hline$\rho_{1}$ & 0.2515183 & 0.9670138 & 0.9345429 & 0.6990442 \\
$D_{1 / 3}$ & 0.2070618 & 0.9649904 & 0.9850216 & 0.6118552 \\
$D_{1}$ & 0.2823364 & 0.9672218 & 0.9353575 & 0.7222155 \\
\hline
\end{tabular}

\section{Concluding remarks}

Different robust alternatives to summarize the central tendency of a random fuzzy number have been empirically compared in terms of their maximum asymptotic bias. This simulation-based study complements the comparative analysis of their finite sample behaviour published previously. In such an analysis it was concluded that, although there is no uniformly most appropriate location estimate, the results seem to be more influenced by the distributions considered for the generation of the sample data than by other aspects such as the sample size. By empirically comparing maxbias curves and asymptotic biases, we have come to the conclusion that the behaviour of the fuzzy trimmed mean with $\beta=0.5$ and the Hampel fuzzy M-estimator of location should be highlighted among the fuzzy-valued location measures. Indeed, the performance of the fuzzy trimmed means with a high trimming level and the Hampel fuzzy M-estimator of location was already commended before because they yielded the best solutions in many situations considered in the previously published comparative analysis of the finite sample behaviour of fuzzy number-valued location measures [9]. The fuzzy trimmed means with $\beta=0.5$ often yields the lowest asymptotic bias in the developed simulation studies (e.g., when $C_{D} \geq 2$ ), but their high asymptotic variance, in contrast to the minimum asymptotic variance achieved by fuzzy M-estimators of location, is a high price to pay. It should be also mentioned that the wabl/ldev/rdev median is the third preferred option based on our empirical results. 
In further research, it would be desirable to extend these simulation studies and cover more situations, including different distributions to generate the contaminated and non contaminated data, such that the current lower bounds for the maximal asymptotic biases become sharper. Furthermore, the lack of realistic models for the distribution of a random fuzzy number makes it difficult to evaluate whether a bias is large or not. Future work should be focused on clarifying this issue, and analyzing whether one should prefer an estimator with a low bias most of the time, but which could have a large bias in a particular situation (such as the fuzzy trimmed mean with $\beta=0.5$ ) or an estimator which has a considerable bias in most situations.

\section{Acknowledgment}

The authors are grateful to the reviewers and Editor handling this paper for their insightful comments and suggestions. This research has been partially supported by the Principality of Asturias/FEDER Grant GRUPINIDI2018-000132 and the Spanish Ministry of Cience, Innovation and Universities Grant MTM2015-63971-P. Their support is gratefully acknowledged.

\section{References}

[1] S. De la Rosa de Sáa, M.A. Gil, G. González-Rodríguez, M.T. López, M.A. Lubiano, Fuzzy rating scale-based questionnaires and their statistical analysis, IEEE Trans. Fuzzy Syst. 23 (1) (2015) 111-126.

[2] M.L. Puri, D.A. Ralescu, Fuzzy random variables, J. Math. Anal. Appl. 114 (1986) 409-422.

[3] B. Sinova, S. Van Aelst, Advantages of M-estimators of location for fuzzy numbers based on Tukey's biweight loss function, Int. J. Appr. Reas. 93 (2018) 219-237.

[4] B. Sinova, M. A. Gil, S. Van Aelst, M-estimates of location for the robust central tendency of fuzzy data, IEEE Trans. Fuzzy Syst. 24 (4) (2016) 945-956.

[5] B. Sinova, S. Pérez-Fernández, M. Montenegro, The wabl/ldev/rdev median of a random fuzzy number and statistical properties, in: P. Grzegorzewski, M. Gagolewski, O. Hryniewicz, M. A. Gil (Eds.), Strengthening Links Between Data Analysis and Soft Computing, Vol. 315 of 
Advances in Intelligent Systems and Computing, Springer, Heidelberg, 2015, pp. 143-150.

[6] A. Colubi, G. González-Rodríguez, Fuzziness in data analysis: Towards accuracy and robustness, Fuzzy Sets Syst. 281 (2015) 260-271.

[7] B. Sinova, S. de la Rosa de Sáa, M.A. Gil, A generalized L1-type metric between fuzzy numbers for an approach to central tendency of fuzzy data, Inf. Sci. 242 (2013) 22-34.

[8] B. Sinova, M.A. Gil, A. Colubi, S. Van Aelst, The median of a random fuzzy number. The 1-norm distance approach, Fuzzy Sets Syst. 200 (2012) 99-115.

[9] B. Sinova, S. Van Aelst, Empirical Comparison of the Performance of Location Estimates of Fuzzy Number-Valued Data, in: S. Destercke, T. Denoeux, M. A. Gil, P. Grzegorzewski, O. Hryniewicz (Eds.), Uncertainty Modelling in Data Science, Vol. 832 of Advances in Intelligent Systems and Computing, Springer, Switzerland, 2019, pp. 191-199.

[10] A. Blanco-Fernández, R. M. Casals, A. Colubi, N. Corral, M. GarcíaBárzana, M. A. Gil, G. González-Rodríguez, M. T. López, M. A. Lubiano, M. Montenegro, A. B. Ramos-Guajardo, S. de la Rosa de Sáa, B. Sinova, A distance-based statistical analysis of fuzzy number-valued data, Int. J. Approx. Reason. 55 (2014) 1487-1501.

[11] A. Blanco-Fernández, R. M. Casals, A. Colubi, N. Corral, M. GarcíaBárzana, M. A. Gil, G. González-Rodríguez, M. T. López, M. A. Lubiano, M. Montenegro, A. B. Ramos-Guajardo, S. de la Rosa de Sáa, B. Sinova, Rejoinder on "a distance-based statistical analysis of fuzzy number-valued data", Int. J. Approx. Reason. 55 (2014) 1601-1605.

[12] M. Montenegro, M. R. Casals, M. A. Lubiano, M. A. Gil, Two-sample hypothesis tests of means of a fuzzy random variable, Inf. Sci. 133 (2001) 89-100.

[13] C. Bertoluzza, N. Corral, A. Salas, On a new class of distances between fuzzy numbers, Math \& Soft Comput. 2 (1995) 71-84.

[14] M. L. Puri, D. A. Ralescu, Fuzzy random variables, J. Math. Anal. Appl. 114 (1986) 409-422. 
[15] M. L. Puri, D. A. Ralescu, The concept of normality for fuzzy random variables, Ann. Probab. 11 (1985) 1373-1379.

[16] M. A. Gil, A. Colubi, P. Terán, Random fuzzy sets: why, when, how, BEIO 30 (1) (2013) 5-29.

[17] R. A. Maronna, D. R. Martin, V. J. Yohai, John Wiley \& Sons, 2006.

[18] P. J. Huber, Robust Statistics, J. Wiley \& Sons, New York, 1981.

[19] P. J. Huber, Robust estimation of a location parameter, Ann. Math. Statist. 35 (1964) 73-101.

[20] J. R. Berrendero, R. H. Zamar, Maximum bias curves for robust regression with non-elliptical regressors, Ann. Statist. 29 (1) (2001) 224-251.

[21] J. A. Cuesta-Albertos, R. Fraiman, Impartial trimmed means for functional data, in: R. Y. Liu, R. Serfling, D. L. Souvaine (Eds.), Data Depth: Robust Multivariate Statistical Analysis, Computational Geometry and Applications, Vol. 72 of DIMACS Series, Amer. Math. Soc., USA, 2006, pp. 121-145.

[22] A. Cuesta-Albertos, R. Fraiman, Impartial trimmed k-means for functional data, Comp. Stat. Data Anal. 51 (10) (2007) 4864-4877.

[23] A. Colubi, J. S. Domínguez-Menchero, M. López-Díaz, M. A. Gil, A generalized strong law of large numbers, Probab. Theory Rel. 114 (1999) 401-417.

[24] P. Diamond, P. Kloeden, Metric spaces of fuzzy sets, Fuzzy Sets Syst. 35 (1990) 241-249.

[25] M. A. Lubiano, A. Salas, M. A. Gil, A hypothesis testing-based discussion on the sensitivity of means of fuzzy data with respect to data shape, Fuzzy Sets Syst. 328 (2017) 54-69.

[26] J. S. Kim, C. D. Scott, Robust kernel density estimation, J. Mach. Learn. Res. 13 (2012) 2529-2565. 